\title{
Nobiletin ameliorates isoflurane-induced cognitive impairment via antioxidant, anti-inflammatory and anti-apoptotic effects in aging rats
}

\author{
JUNYING BI ${ }^{1,2}$, HAIYAN ZHANG ${ }^{3}$, JING LU ${ }^{2}$ and WEIFU LEI ${ }^{1}$ \\ ${ }^{1}$ Department of Anesthesiology, Qilu Hospital, Shandong University, Jinan, Shandong 250012; \\ ${ }^{2}$ Department of Anesthesiology; ${ }^{3}$ Gynaecology Ward-1, Linyi People's Hospital, Linyi, Shandong 276000, P.R. China
}

Received August 7, 2015; Accepted August 22, 2016

DOI: $10.3892 / \mathrm{mmr} .2016 .5919$

\begin{abstract}
A recent study reported that nobiletin is an active ingredient in Fructus Aurantii immaturus and Pericarpium Citri Reticulatae, which may be capable of preventing ischemic stroke. Therefore, the present study aimed to determine the neuroprotective effects of nobiletin, and to evaluate whether it could ameliorate isoflurane-induced cognitive impairment via antioxidant, anti-inflammatory and anti-apoptotic effects in aging rats. Male Sprague-Dawley rats (age, 18 months) were used to analyze the neuroprotective effects of nobiletin. Morris water maze test was used to determine cognitive competence. Enzyme-linked immunosorbent assay and western blot analysis were also used to quantify nuclear factor $-\kappa \mathrm{B}$, tumor necrosis factor (TNF)- $\alpha$, IL-1 $\beta$, IL-6, glutathione, (GSH), GSH-peroxidase, superoxide dismutase and malondialdehyde concentration and relevant protein expression levels Cognitive competence was increased in isoflurane-treated rats following treatment with nobiletin. In addition, as expected, nobiletin exerted antioxidant, anti-inflammatory and anti-apoptotic effects on isoflurane-induced cognitive impairment in aging rats. Treatment with nobiletin induced the activation of phosphorylated (p)-Akt, p-cAMP response element binding protein (CREB) and brain-derived neurotrophic factor (BDNF) protein expression and reduced the levels of B-cell lymphoma 2 -associated $\mathrm{X}$ protein $(\mathrm{Bax})$ in isoflurane-induced rats. In conclusion, the present study demonstrated that nobiletin may ameliorate isoflurane-induced cognitive impairment through antioxidant, anti-inflammatory and anti-apoptotic effects via modulation of Akt, Bax, p-CREB and BDNF in aging rats. These findings provide support for the molecular
\end{abstract}

Correspondence to: Dr Weifu Lei, Department of Anesthesiology, Qilu Hospital, Shandong University, 107 Wenhua Xi Road, Jinan, Shandong 250012, P.R. China

E-mail:weifulei@yeah.net

Key words: nobiletin, isoflurane, cognitive impairment, aging rat mechanisms underlying the effects of nobiletin treatment on isoflurane-induced damage.

\section{Introduction}

Inhalational anesthetics have been used in clinical practice for $>170$ years; they have been extensively applied due to their numerous advantages, including anesthetic efficacy and safety, and the ease at which they can be used to regulate anesthetic depth (1). In the United States, the annual number of cases where general anesthesia is used is $\sim 40,000,000$, and anesthesia has an important role in the safety and success of surgery. In recent years, inhalational anesthetics have been reported to protect against ischemic injury in the cardiovascular system, brain, kidney and other important organs (2); and the potential clinical application of these drugs may be wider than at present. However, further studies are required on how inhalational anesthetics induce unconsciousness, immobilization and analgesia, and other general anesthetic effects. In addition, it is necessary to determine how inhaled anesthesia induces intraoperative awareness and postoperative agitation, nausea and vomiting, and postoperative cognitive decline (POCD), and how it impacts the intelligence and central nervous system development of young children (3). Such issues have attracted extensive clinical attention. Clarification of the aforementioned pathogenetic mechanisms not only has far-reaching significance on revealing the underlying mechanism of general anesthesia, but may also be used to guide clinical medication, improve the safety of clinical anesthesia, and for the development of novel general anesthetics and antagonists (4). Understanding the underlying mechanisms of anesthesia is conducive to understanding the neuroscience of consciousness, memory, perception, movement and awakening (5).

The pathogenetic mechanism of POCD is complex, and research regarding the mechanism is diverse. It has previously been indicated that halothane can induce cognitive impairment (6). In addition, it has been reported that increased hippocampal inflammatory cytokine expression may induce transitory cognitive impairment (7). In mice with an interleukin (IL)-1 receptor knockout, peripheral operation-induced hippocampal neuronal inflammation and IL-1 $\beta$-induced autoimmune response are associated with memory impairment. 
In addition, continuous excessive expression of hippocampal IL-1 $\beta$ may lead to contextual memory and spatial memory impairment of mice (8).

Nobiletin (Fig. 1) is a type of polymethoxyflavonoid, which is predominantly found in Pericarpium Citri Reticulatae, a traditional Chinese medicine (9). Nobiletin exists in the pericarp of citrus fruits, and exerts extensive physiological effects that are beneficial to health. Therefore, nobiletin has attracted clinical attention (10). As well as its anti-inflammatory, antioxidant and anticancer activity, nobiletin has roles in anti-atherosclerosis, reducing blood glucose levels, liver protection and nerve nutrition (11). Previous studies have demonstrated that rat hippocampal neurons cultivated in nobiletin may enhance the signal channel of protein kinase A/extracellular signal-regulated kinase/cAMP response element binding protein (CREB) to alleviate memory deterioration caused by $\mathrm{P}$-amyloid protein in mice with Alzheimer's disease model and amyloid precursor protein genetically modified, and to improve learning and memory disorder caused by cerebral ischemia $(12,13)$. The aim of the present study was to determine the neuroprotective effects of nobiletin, and to evaluate whether it could ameliorate cognitive impairment via antioxidant, anti-inflammatory and anti-apoptotic effects in isoflurane-treated aging rats.

\section{Materials and methods}

Ethical approval and experimental animals. The animal protocol was approved by the Standing Committee on Animals at Shandong University (Jinan, China). Male Sprague-Dawley rats (age, 18 months) were acquired from the Institute of Experimental Animals, Shandong University. All rats were given ad libitum access to food and water, and were maintained under controlled laboratory conditions: 12/12 h light/dark cycle, $55 \pm 5 \%$ humidity and $23 \pm 2^{\circ} \mathrm{C}$.

Rat model of isoflurane-induced cognitive impairment. All aging rats were allowed to acclimate to the environment for 1 week, and were then randomly separated into four groups: i) Isoflurane group $(n=20)$; ii) nobiletin (10) group $(n=20)$; iii) nobiletin (25) group ( $n=20)$; and iv) sham group $(n=10)$. Rats in the isoflurane and nobiletin (10) and (25) groups were administered $1.4 \%$ isoflurane in a $100 \%$ oxygen environment for $2 \mathrm{~h}$ in an anesthetization chamber. Rats were maintained at $37.5 \pm 0.5^{\circ} \mathrm{C}$ using a heating pad. In the nobiletin (10) and (25) groups, the rats were intraperitoneally injected with nobiletin (Sigma-Aldrich; Merck Millipore, Darmstadt, Germany) at 10 or $25 \mathrm{mg} / \mathrm{kg} /$ day for 3 days, respectively (1).

Behavioral testing. A circular pool (150x50 cm; depth, $31 \mathrm{~cm}$; $22 \pm 1^{\circ} \mathrm{C}$ ) was used to conduct the Morris water maze test. The pool area was divided into SW, NW, SE and NE quadrants. A transparent platform (diameter, $9 \mathrm{~cm}$ ) $2 \mathrm{~cm}$ below water level was placed in the center of the SW quadrant. All rats were trained to find the escape platform in order to test reference memory. The rats underwent eight trials for 30 min every day. The maximum swimming time was set at $120 \mathrm{sec}$ and ended with the animal finding the platform. Each rat was gently guided to the platform, which remained for $30 \mathrm{sec}$, if the time limit was exceeded. Escape latency, path length (length taken

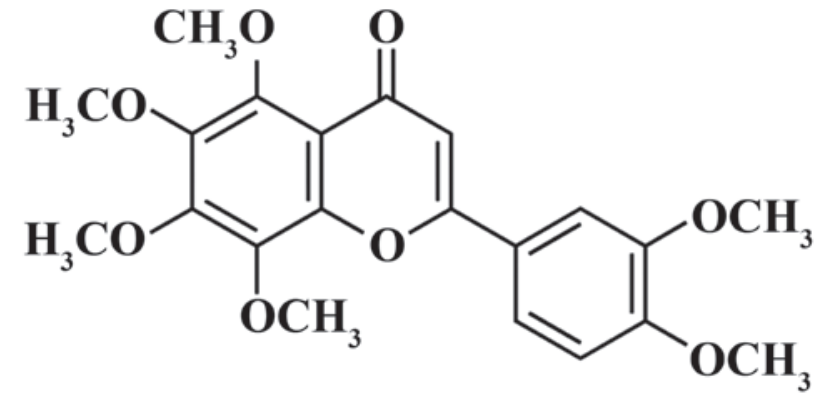

Figure 1. Chemical structure of nobiletin.

to reach the platform) and swimming speed were all analyzed. After the last reference memory test, the spatial probe test was performed. Briefly, the rats were placed into the water and were allowed to swim for $120 \mathrm{sec}$. Path length and time spent in the target quadrant were recorded. The number of platform crossings were analyzed using Actimetrics motion detection software for the Morris Water Maze (Actimetrics Software, Evanston, IL, USA).

Enzyme-linked immunosorbent assay (ELISA). Whole blood samples were immediately collected by centrifugation at $4,000 \times \mathrm{g}$ for $10 \mathrm{mins}$ at $4^{\circ} \mathrm{C}$ to measure serum nuclear factor (NF)- $\kappa \mathrm{B}$, tumor necrosis factor (TNF)- $\alpha$, IL- $1 \beta$ and IL- 6 concentration using ELISA kits obtained from Nanjing Jiancheng Bioengineering Institute (Nanjing, China). Glutathione (GSH; Nanjing Jiancheng Bioengineering Institute), GSH peroxidase (GSH-PX; Nanjing Jiancheng Bioengineering Institute), superoxide dismutase (SOD; Elabscience Biotechnology Co. Ltd., Wuhan, China) and malondialdehyde (MDA; Elabscience Biotechnology Co. Ltd.) concentrations were also determined using ELISA kits , according to the manufacturers' protocols.

Western blot analysis. The rats were anesthetized with $30 \mathrm{mg} / \mathrm{kg}$ pentobarbital sodium and then sacrificed by decollation. Hippocampi were then immediately removed and maintained in liquid nitrogen. Frozen hippocampi were subsequently weighed, and $\sim 50 \mathrm{mg}$ tissue was homogenized in RIPA buffer with protease inhibitors (Beyotime Insititute of Biotechnology, Haimen, China). The homogenates were centrifuged at $12,000 \times \mathrm{g}$ for $10 \mathrm{~min}$ at $4^{\circ} \mathrm{C}$, and the supernatant was collected in order to measure protein content using bicinchoninic acid assay kit according to the manufacturer's protocol (Beyotime Insititute of Biotechnology). Equal amounts of protein samples $(50 \mu \mathrm{g})$ were separated by $10-12 \%$ sodium dodecyl sulfate-polyacrylamide gel electrophoresis and were transferred to polyvinylidene fluoride membranes (EMD Millipore, Billerica, MA, USA). After blocking with $5 \%$ skimmed milk in Tris-buffered saline $1 \%$ Tween-20 for $1 \mathrm{~h}$, the membranes were probed with the following primary antibodies: Anti-B-cell lymphoma 2-associated X protein (Bax; cat. no. sc-6236; 1:500; Santa Cruz Biotechnology, Inc., Dallas, TX, USA), anti-phosphorylated (p)-Akt (cat. no. sc-135650; 1:500; Santa Cruz Biotechnology, Inc.), anti-p-CREB (cat. no. sc-81486; 1:500; Santa Cruz Biotechnology, Inc.), anti-brain-derived neurotrophic factor 

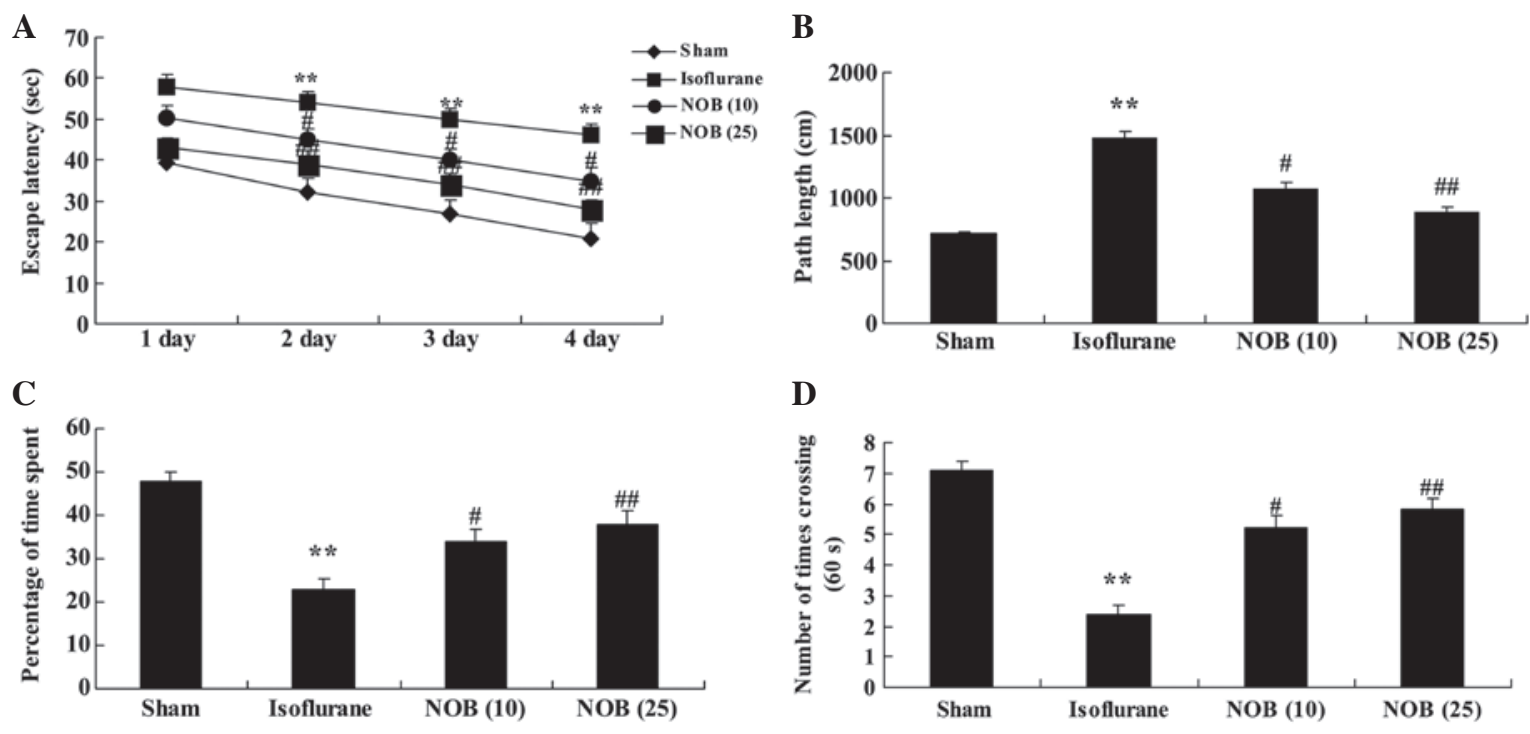

Figure 2. Memory testing of isoflurane-treated rats. (A) Escape latency, (B) path length, (C) time spent in target quadrant and (D) number of times the rat crossed the platform. ${ }^{* *} \mathrm{P}<0.01$ compared with the sham group; ${ }^{\prime \prime} \mathrm{P}<0.05,{ }^{\# \prime \prime} \mathrm{P}<0.01$ compared with the isoflurane group. NOB (10), $10 \mathrm{mg} / \mathrm{kg}$ nobiletin; NOB (25), $25 \mathrm{mg} / \mathrm{kg}$ nobiletin.
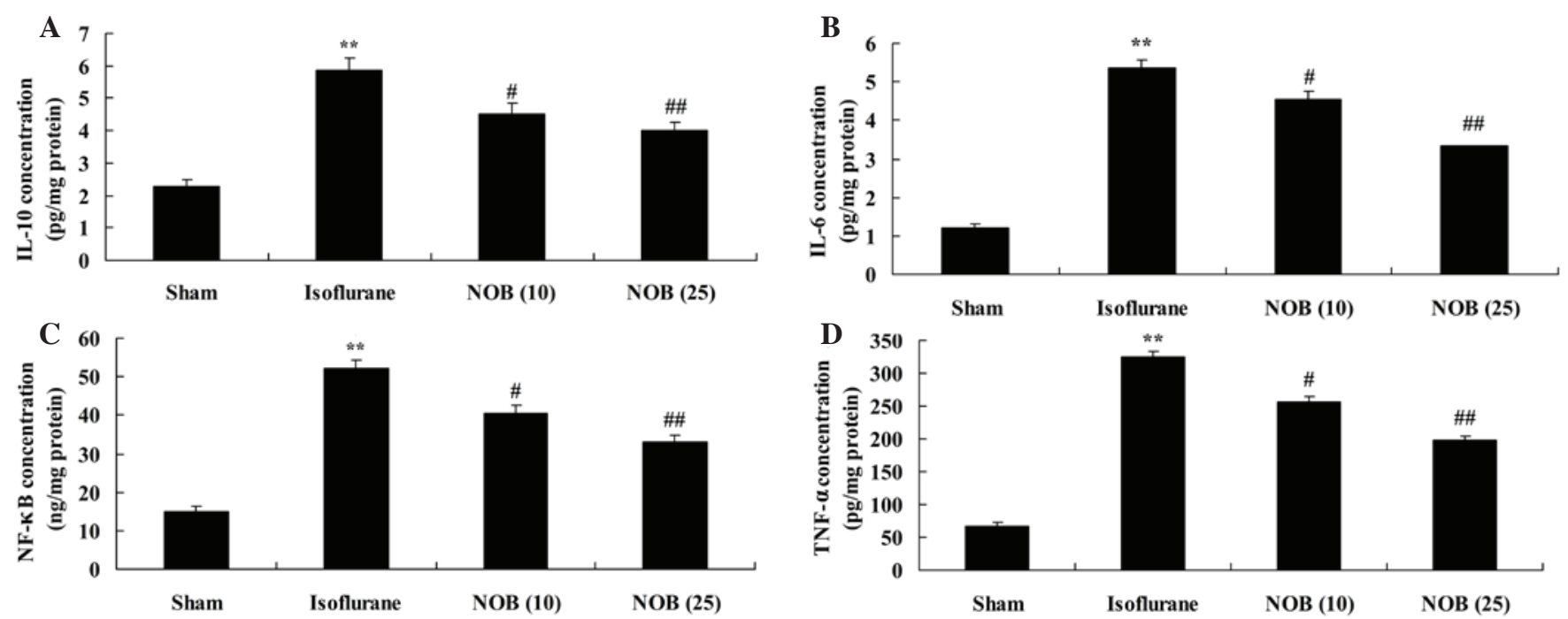

Figure 3. Antioxidant effects of nobiletin in isoflurane-treated rats. (A) Glutathione peroxidase (GSH-PX), (B) GSH, (C) superoxide dismutase (SOD) and (D) malondialdehyde (MDA) concentrations. ${ }^{* *} \mathrm{P}<0.01$ compared with the sham group; ${ }^{\#} \mathrm{P}<0.05,{ }^{\# /} \mathrm{P}<0.01$ compared with the isoflurane group. NOB (10), $10 \mathrm{mg} / \mathrm{kg}$ nobiletin; NOB (25), $25 \mathrm{mg} / \mathrm{kg}$ nobiletin.

(BDNF; cat. no. sc-20981; 1:500; Santa Cruz Biotechnology, Inc.) and anti- $\beta$-actin (cat. no. sc-130656; $1: 500$; Santa Cruz Biotechnology, Inc.) overnight at $4^{\circ} \mathrm{C}$. Proteins were detected using horseradish peroxidase-conjugated anti-rabbit secondary antibodies (cat. no. sc-2054; 1:1,000; Santa Cruz Biotechnology, Inc.) at room temperature for $1 \mathrm{~h}$. Blots were visualized using an enhanced chemiluminescence kit (Santa Cruz Biotechnology, Inc.). The membranes were analyzed using Image-Pro Plus 6.0 software (Media Cybernetics, Inc., Rockville, MD, USA).

Statistical analysis. Results are presented as the mean \pm standard deviation and were analyzed using one-way analysis of variance, followed by Tukey post-hoc multiple comparisons test using GraphPad Prism version 4.0 (GraphPad Software,
Inc., La Jolla, CA, USA). $\mathrm{P}<0.05$ was considered to indicate a statistically significant difference.

\section{Results}

Memory testing. To examine whether nobiletin exerted neuroprotective effects on isoflurane-induced cognitive impairment, memory testing was conducted. As shown in Fig. 2A and B, treatment with isoflurane induced an increase in escape latency and path length compared with in the sham group. Treatment with nobiletin effectively decreased the escape latency and path length in a dose-dependent manner. In addition, there was a significant reduction in time spent in the target quadrant and the number of times the rat crossed the platform in the isoflurane model group compared with in the sham group (Fig. $2 \mathrm{C}$ 

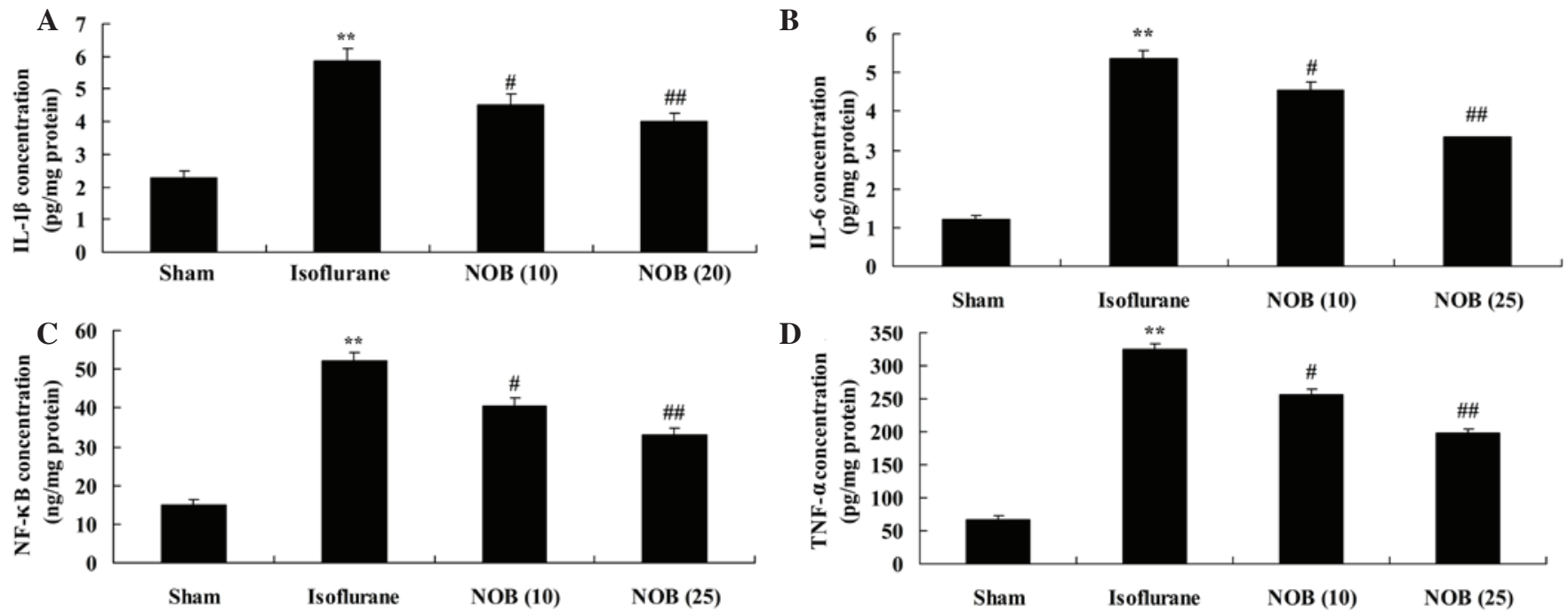

Figure 4. Anti-inflammatory effects of nobiletin in isoflurane-treated rats. (A) Interleukin (IL)-1 $\beta$, (B) IL-6, (C) nuclear factor (NF)- $\mathrm{kB}$ and (D) tumor necrosis factor (TNF)- $\alpha$ concentrations. ${ }^{* *} \mathrm{P}<0.01$ compared with the sham group; ${ }^{\#} \mathrm{P}<0.05,{ }^{\# \#} \mathrm{P}<0.01$ compared with the isoflurane group. NOB (10), $10 \mathrm{mg} / \mathrm{kg}$ nobiletin; NOB (25), $25 \mathrm{mg} / \mathrm{kg}$ nobiletin.
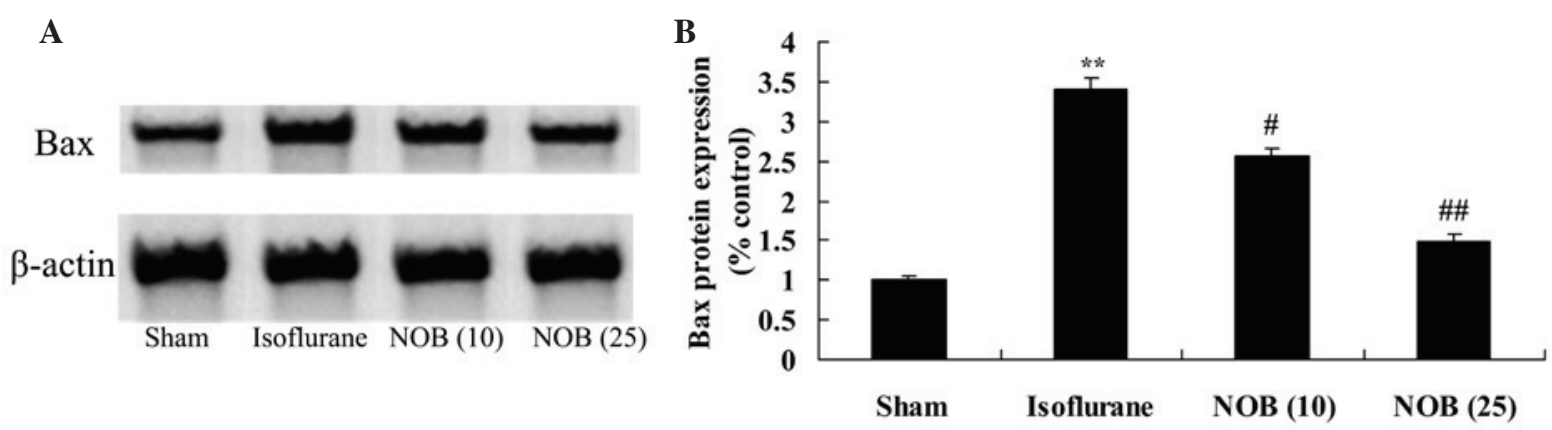

Figure 5. (A) Protein expression levels of B-cell lymphoma 2-associated X protein (Bax) were detected using western blot analysis. (B) Semi-quantitative analysis of Bax protein expression in isoflurane-treated rats. ${ }^{* *} \mathrm{P}<0.01$ compared with the sham group; ${ }^{~} \mathrm{P}<0.05,{ }^{\# \#} \mathrm{P}<0.01$ compared with the isoflurane group. NOB (10), $10 \mathrm{mg} / \mathrm{kg}$ nobiletin; NOB (25), $25 \mathrm{mg} / \mathrm{kg}$ nobiletin.

A

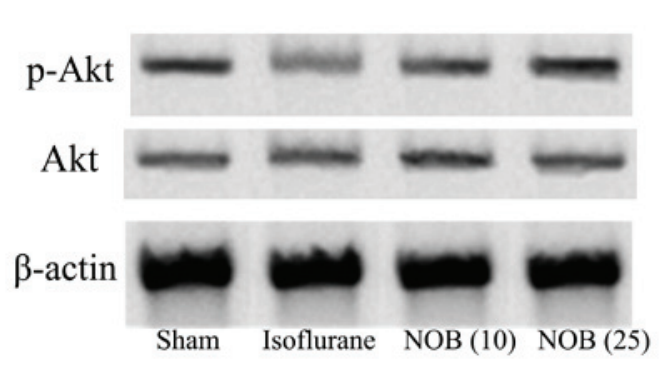

B

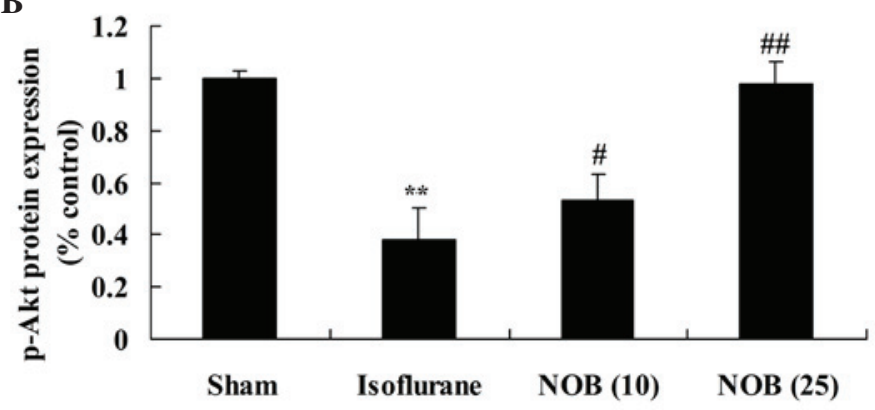

Figure 6. (A) Protein expression levels of phosphorylated (p)-Akt were detected by western blot analysis. (B) Semi-quantitative analysis of p-Akt protein expression in isoflurane-treated rats. ${ }^{* *} \mathrm{P}<0.01$ compared with the sham group; ${ }^{\mathrm{P}} \mathrm{P}<0.05,{ }^{\# \#} \mathrm{P}<0.01$ compared with the isoflurane group. NOB (10), $10 \mathrm{mg} / \mathrm{kg}$ nobiletin; NOB (25), $25 \mathrm{mg} / \mathrm{kg}$ nobiletin.

and D). However, treatment with nobiletin significantly increased time spent in the target quadrant and the number of times the rat crossed the platform (Fig. 2C and D).

Antioxidant effects. To examine whether nobiletin exerted neuroprotective effects on isoflurane-induced oxidative damage, GSH-PX, GSH, SOD and MDA concentrations were analyzed using ELISA kits. As shown in Fig. 3A-D, isoflurane inhibited GSH-PX, GSH and SOD concentrations, whereas MDA concentration was increased in the isoflurane group compared with in the sham group. Conversely, treatment with nobiletin significantly increased GSH-PX, GSH and SOD concentrations, and reduced MDA concentration in isoflurane-treated rats (Fig. 3A-D). 
A

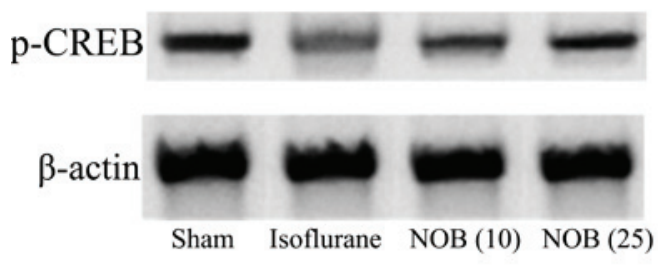

B

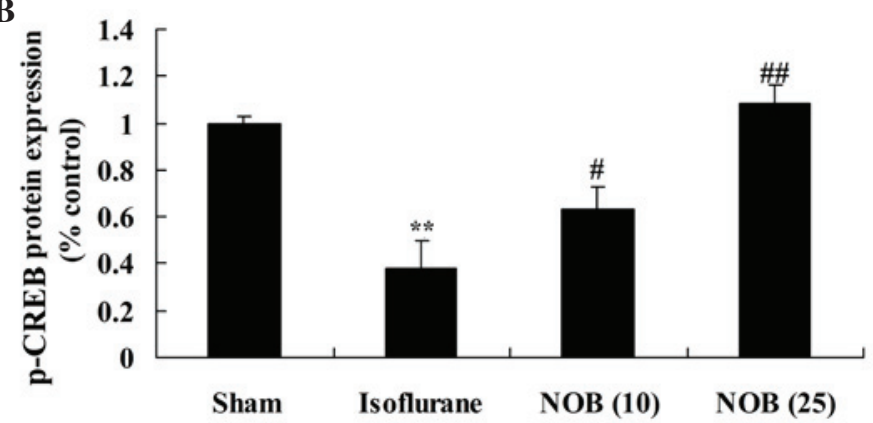

Figure 7. (A) Protein expression levels of phosphorylated-cAMP response element binding protein (p-CREB) were detected by western blot analysis. (B) Semi-quantitiative analysis of $\mathrm{p}$-CREB protein expression in isoflurane-treated rats. ${ }^{* *} \mathrm{P}<0.01$ compared with the sham group; ${ }^{\#} \mathrm{P}<0.05$, ${ }^{\# \#} \mathrm{P}<0.01$ compared with the isoflurane group. NOB (10), $10 \mathrm{mg} / \mathrm{kg}$ nobiletin; NOB (25), $25 \mathrm{mg} / \mathrm{kg}$ nobiletin.

A

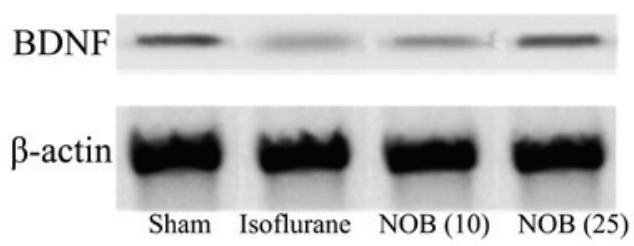

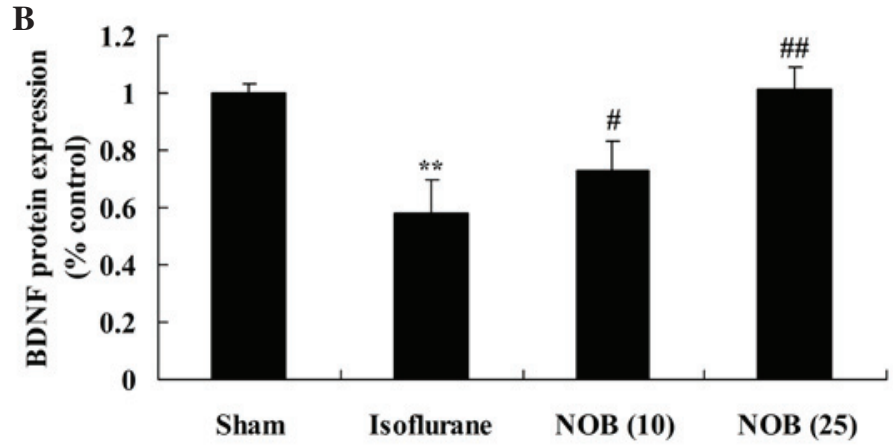

Figure 8. (A) Protein expression levels of brain-derived neurotrophic factor (BDNF) were detected by western blot analysis. (B) Semi-quantitative analysis of BDNF protein expression in isoflurane-treated rats. ${ }^{* *} \mathrm{P}<0.01$ compared with the sham group; ${ }^{~} \mathrm{P}<0.05,{ }^{\# \#} \mathrm{P}<0.01$ compared with the isoflurane group. NOB (10), $10 \mathrm{mg} / \mathrm{kg}$ nobiletin; NOB (25), $25 \mathrm{mg} / \mathrm{kg}$ nobiletin.

Anti-inflammatory effects. Toexamine whether nobiletin exerted neuroprotective effects on isoflurane-induced inflammation, NF- $\kappa B$, TNF- $\alpha$, IL-1 $\beta$ and IL- 6 concentrations were analyzed using ELISA kits. As shown in Fig. 4A-D, isoflurane enhanced NF- $\kappa B$, TNF- $\alpha$, IL- $1 \beta$ and IL-6 concentrations compared with in the sham group. However, treatment with nobiletin significantly reduced NF- $\kappa B$, TNF- $\alpha$, IL- $1 \beta$ and IL- 6 concentrations compared with in the isoflurane-treated rats (Fig. 4A-D).

Protein expression of Bax. The present study assessed the effects of nobiletin on the protein expression levels of Bax by western blot analysis. Bax protein expression was activated by isoflurane compared with in the sham group (Fig. 5). Conversely, treatment with nobiletin significantly suppressed Bax protein expression in isoflurane-treated rats (Fig. 5).

Protein expression of $p$-Akt. The protein expression levels of p-Akt were detected in every group; $\mathrm{p}$-Akt protein expression was decreased following treatment with isoflurane compared with in the sham group (Fig. 6). However, treatment with nobiletin markedly increased p-Akt protein expression compared with in the isoflurane-treated group (Fig. 6).

Protein expression of p-CREB. As shown in Fig. 7, exposure to isoflurane markedly decreased the protein expression levels of p-CREB compared with in the sham group. Treatment with nobiletin increased p-CREB protein expression compared with in the isoflurane-treated group (Fig. 7).
Protein expression of BDNF. To investigate the mechanism underlying the effects of nobiletin on isoflurane-induced cognitive impairment, the protein expression levels of BDNF were detected using western blot analysis. Compared with the sham group, BDNF protein expression was decreased following exposure to isoflurane anesthesia (Fig. 8). Conversely, the protein expression levels of BDNF were increased following treatment with nobiletin (Fig. 8).

\section{Discussion}

The primary cause of POCD is the use of anesthesia, including frequently-used isoflurane, sevoflurane and other inhalational anesthetics, as well as intravenous anesthetics, such as ketamine and propofol (4). Surgical and anesthetic complications can accelerate POCD. POCD induced by isoflurane and other inhalational anesthetics is a complex issue, which has attracted attention from surgeons and anesthesiologists (14). At present, several studies have focused on cognitive impairment induced by isoflurane; however, whether the concentration of frequently-used isoflurane (1-3\%) is able to induce cognitive impairment in patients undergoing surgery, as well as neuronal apoptosis associated with cognitive function, requires further study (15-17). The present study demonstrated that the neuroprotective effects of nobiletin ameliorated isoflurane-induced cognitive impairment in rats.

Proinflammatory and anti-inflammatory cytokines exist in the body; the balance between them is regulated by 
the neuroendocrine and immune systems. An imbalance between proinflammatory and anti-inflammatory cytokines is a significant cause leading to inflammatory injury (18). Among the types of inflammatory damage that affect visceral organs, IL-1 $\beta$ is one of the earliest proinflammatory cytokines. The synergistic effects of IL-1 $\beta$ and TNF- $\alpha$ activate the inflammatory reaction-associated transcription factor, $\mathrm{NF}-\kappa \mathrm{B}$, in immune cells and non-immune cells, which induces the inflammatory cascade reaction, promotes aggregation of granulocytes, and lead to tissue damage (19). The present study demonstrated that nobiletin significantly reduced isoflurane-induced NF- $\mathrm{B}$, TNF- $\alpha$, IL- $1 \beta$ and IL-6 concentrations in rats. Guan et al (20) reported that nobiletin attenuated reactive oxygen species (ROS) production and the expression of nuclear NF- $\mathrm{NB}$ p65 in a rat model of carotid artery injury (20). Jang et al (12) revealed that nobiletin ameliorated scratching behavior via inhibiting the activation of NF- $\kappa \mathrm{B}$, activator protein-1 and p38 in mice.

Dysfunctional energy metabolism and oxidative damage have a role in cognitive impairment, since brain tissue is particularly sensitive to tissue and free radical damage (21). In addition, the brain tissue exerts strict demands on oxygen, and accounts for $20 \%$ of human body oxygen consumption. However, GSH has obviously decreased in brain tissues of isoflurane-induced aged rats (22). Sufficient evidence has revealed the relationship between oxidative stress and cognitive function. Antioxidants are able to reverse the memory impairment of aged rats $(23,24)$. Through lipid metabolite detection and behavioral testing, it has been demonstrated that oxidative damage can aggravate transitory cognitive impairment (25). The learning capacity of mice is higher compared to that of aged rats. SOD is an essential antioxidant in vivo; however, a large amount of SOD may enhance oxidative stress (25). Therefore, decreased SOD levels may 5reflect increasing of oxidative stress levels. MDA is a lipid peroxidation product associated with increased ROS levels. MDA levels can reflect the degree of body lipid peroxidation, or indirectly reflect the degree of tissue and cell damage (22). The present study demonstrated that nobiletin significantly increased GSH-PX, GSH and SOD concentrations, and reduced MDA concentration in isoflurane-treated rats. Lo et al (11) reported that nobiletin inhibits low-density lipoprotein oxidation in THP-1 cells.

The Akt signal transduction pathway is associated with growth, proliferation and regulation of differentiation (26). The Akt pathway promotes survival, and its activation has an important role in nerve cell protection, particularly in hypoxic ischemic neuronal injury. It has recently attracted extensive attention (27). The activation of Akt can promote endothelial cell survival, decrease nerve damage, reduce inflammatory cell death and obstruct damage to thermoregulatory neurons (27). The present study demonstrated that nobiletin markedly increased isoflurane-induced p-Akt protein expression. Zhang et al (28) reported that nobiletin was able to activate $\mathrm{p}$-Akt, $\mathrm{p}$-CREB, BDNF and B-cell lymphoma 2 (Bcl-2) pathways in order to protect against cerebral ischemia in rats.

Apoptosis is a complex type of programmed cell death, which is associated with the regulation of several genes. The Bcl-2 gene family has an important role during the regulatory process. The Bcl-2 family can be divided into two categories, according to effects on the apoptotic process: Proapoptotic genes, including Bax, Bcl-2 antagonist/killer 1 and Bcl-2 associated agonist of cell death; and anti-apoptotic genes, including $\mathrm{Bcl}-2$, Bcl-extra large and $\mathrm{Bcl}-\mathrm{w}$. Interactions between $\mathrm{Bcl}-2$ family proteins may affect cell survival and apoptosis. The interaction between Bax and Bcl-2 is of particular importance. Increased levels of Bax promote cell apoptosis; however, increased levels of Bcl-2 inhibit cell apoptosis. In the present study, nobiletin significantly suppressed Bax protein expression in isoflurane-treated rats. Malik et al (29) suggested that nobiletin may ameliorate cisplatin-induced acute kidney injury by increasing the expression of Bax, and exerting antioxidant, anti-inflammatory and anti-apoptotic effects.

Previous studies regarding learning memory behaviors have indicated that CREB, as 'the third messenger', is important in the long-term memory process (30-32). It is believed, as the optimal nuclear transcription factor, to adapt to external stimulus as well as long term memory. Both the cortical neuronal at plasticity forming process and hippocampal neuron under long range increase stimulation and memory training task, CREB phosphorylation and CRE reporter gene expression can be detected. However, in mice with decreased spatial memory ability, the phosphorylation level of CREB in the hippocampus has been reported to be markedly decreased (33). In the present study, the protein expression levels of CREB were enhanced following treatment with nobiletin, as compared with in the isoflurane-induced model group. Zhang et al (28) demonstrated that nobiletin activated p-Akt, CREB, BDNF and Bcl-2 pathways for protection against cerebral ischemia in rats.

BDNF is an important member of the glial cell line-derived neurotrophic factor family, which is predominantly distributed throughout the central nervous system, particularly the hippocampal area. BDNF exerts differentiative, proliferative and nutritive effects on several types of neuron (34). In addition, it exerts promoting effects on the synthesis of neurotransmitter and neurotrophic factors, and is closely associated with learning, memory and cognitive processes (35). At present, it is believed that the occurrence of POCD is caused by neuronal necrosis and apoptosis via multiple pathways. Previous animal experiments have indicated that at the onset of POCD, the expression of BDNF is markedly enhanced, which may significantly alleviate ischemic brain injury, inhibit neuronal death, and exert neuroprotective effects $(35,36)$. In the present study, the protein expression levels of BDNF were increased following treatment of isoflurane-treated rats with nobiletin. Zhang et al (28) reported that nobiletin activated p-Akt, p-CREB, BDNF and $\mathrm{Bcl}-2$ pathways in order to protect against cerebral ischemia in rats. In addition, Li et al (37) suggested that nobiletin may ameliorate hippocampal deficits via BDNF.

In conclusion, the present study demonstrated that nobiletin exerts neuroprotective effects and ameliorates isoflurane-induced cognitive impairment in aging rats. Nobiletin exerted antioxidant, anti-inflammatory and anti-apoptotic effects via the p-Akt, p-CREB, BDNF and Bcl-2 signaling pathways.

\section{References}

1. Zhang Y, Dong Y, Xu Z and Xie Z: Propofol and magnesium attenuate isoflurane-induced caspase-3 activation via inhibiting mitochondrial permeability transition pore. Med Gas Res 2: 20, 2012. 
2. Wiese AJ, Brosnan RJ and Barter LS: Effects of acetylcholinesterase inhibition on quality of recovery from isoflurane-induced anesthesia in horses. Am J Vet Res 75: 223-230, 2014

3. Cheng B, Zhang Y, Wang A, Dong Y and Xie Z: Vitamin C attenuates Isoflurane-Induced Caspase-3 activation and cognitive impairment. Mol Neurobiol 52: 1580-1589, 2015.

4. Ni C, Li Z, Qian M, Zhou Y, Wang J and Guo X: Isoflurane induced cognitive impairment in aged rats through hippocampal calcineurin/NFAT signaling. Biochem Biophys Res Commun 460: 889-895, 2015.

5. Sanders RD, Xu J, Shu Y, Januszewski A, Halder S, Fidalgo A, Sun P, Hossain M, Ma D and Maze M: Dexmedetomidine attenuates isoflurane-induced neurocognitive impairment in neonatal rats. Anesthesiology 110: 1077-1085, 2009.

6. Scuteri A and Wang H: Pulse wave velocity as a marker of cognitive impairment in the elderly. J Alzheimers Dis 42 (Suppl 4): S401-S410, 2014

7. Shu Y, Zhou Z, Wan Y, Sanders RD, Li M, Pac-Soo CK, Maze M and Ma D: Nociceptive stimuli enhance anesthetic-induced neuroapoptosis in the rat developing brain. Neurobiol Dis 45: 743-750, 2012

8. Cao L, Li L, Lin D and Zuo Z: Isoflurane induces learning impairment that is mediated by interleukin $1 \beta$ in rodents. PLoS One 7: e51431, 2012

9. Nakajima A, Ohizumi Y and Yamada K: Anti-dementia activity of Nobiletin, a Citrus Flavonoid: A review of animal studies. Clin Psychopharmacol Neurosci 12: 75-82, 2014.

10. Yamakuni T, Nakajima A and Ohizumi Y: Pharmacological action of nobiletin, a component of AURANTII NOBILIS PERICARPIUM with anti-dementia activity and its application for development of functional foods. Nihon Yakurigaku Zasshi 132: 155-159, 2008.

11. Lo YH, Pan MH, Li S, Yen JH, Kou MC, Ho CT and Wu MJ: Nobiletin metabolite, 3',4'-dihydroxy-5,6,7,8-tetramethoxyflavone, inhibits LDL oxidation and down-regulates scavenger receptor expression and activity in THP-1 cells. Biochim Biophys Acta 1801: 114-126, 2010

12. Jang SE, Ryu KR, Park SH, Chung S, Teruya Y, Han MJ, Woo JT and Kim DH: Nobiletin and tangeretin ameliorate scratching behavior in mice by inhibiting the action of histamine and the activation of NF- $\kappa$ B, AP-1 and p38. Int Immunopharmacol 17: 502-507, 2013.

13. Al Rahim M, Nakajima A, Saigusa D, Tetsu N, Maruyama Y, Shibuya M, Yamakoshi H, Tomioka Y, Iwabuchi Y, Ohizumi Y and Yamakuni T: 4'-Demethylnobiletin, a bioactive metabolite of nobiletin enhancing PKA/ERK/CREB signaling, rescues learning impairment associated with NMDA receptor antagonism via stimulation of the ERK cascade. Biochemistry 48: 7713-7721, 2009.

14. Kupershmidt L, Amit T, Bar-Am O, Weinreb O and Youdim MB: Multi-target, neuroprotective and neurorestorative M30 improves cognitive impairment and reduces Alzheimer's-like neuropathology and age-related alterations in mice. Mol Neurobiol 46 217-220, 2012

15. Liang G, Ward C, Peng J, Zhao Y, Huang B and Wei H: Isoflurane causes greater neurodegeneration than an equivalent exposure of sevoflurane in the developing brain of neonatal mice. Anesthesiology 112: 1325-1334, 2010.

16. Yang N,Liang Y, Yang P, Wang W,Zhang X and Wang J: TNF-alpha receptor antagonist attenuates isoflurane-induced cognitive impairment in aged rats. Exp Ther Med 12: 463-468, 2016.

17. Wang X, Zhao B and Li X: Dexmedetomidine attenuates isoflurane-induced cognitive impairment through antioxidant, anti-inflammatory and anti-apoptosis in aging rat. Int J Clin Exp Med 8: 17281-17288, 2015

18. Kim YK, Na KS, Myint AM and Leonard BE: The role of pro-inflammatory cytokines in neuroinflammation, neurogenesis and the neuroendocrine system in major depression. Prog Neuropsychopharmacol Biol Psychiatry 64: 277-284, 2016.

19. Castanon N, Lasselin J and Capuron L: Neuropsychiatric comorbidity in obesity: Role of inflammatory processes. Front Endocrinol (Lausanne) 5: 74, 2014.
20. Guan S, Tang Q, Liu W, Zhu R and Li B: Nobiletin inhibits PDGF-BB-induced vascular smooth muscle cell proliferation and migration and attenuates neointimal hyperplasia in a rat carotid artery injury model. Drug Dev Res 75: 489-496, 2014.

21. Golechha M, Sarangal V, Bhatia J, Chaudhry U, Saluja D and Arya DS: Naringin ameliorates pentylenetetrazol-induced seizures and associated oxidative stress, inflammation and cognitive impairment in rats: Possible mechanisms of neuroprotection. Epilepsy Behav 41: 98-102, 2014.

22. Liu GS, Zhang ZS, Yang B and He W: Resveratrol attenuates oxidative damage and ameliorates cognitive impairment in the brain of senescence-accelerated mice. Life Sci 91: 872-877, 2012.

23. Leite MR, Wilhelm EA, Jesse CR, Brandao R and Nogueira $\mathrm{CW}$ : Protective effect of caffeine and a selective A2A receptor antagonist on impairment of memory and oxidative stress of aged rats. Exp Gerontol 46: 309-315, 2011.

24. Haider S, Saleem S, Perveen T, et al: Age-related learning and memory deficits in rats: role of altered brain neurotransmitters, acetylcholinesterase activity and changes in antioxidant defense system. Age (Dordr) 36: 9653, 2014

25. Wang C, He L, Yan M, Zheng GY and Liu XY: Effects of polyprenols from pine needles of Pinus massoniana on ameliorating cognitive impairment in a D-galactose-induced mouse model. Age (Dordr) 36: 9676, 2014

26. $\mathrm{Li} \mathrm{T}$ and Wang $\mathrm{G}$ : Computer-aided targeting of the PI3K/Akt/mTOR pathway: Toxicity reduction and therapeutic opportunities. Int J Mol Sci 15: 18856-18891, 2014.

27. Lu W, Xu Z, Zhang M and Zuo Y: MiR-19a promotes epithelial-mesenchymal transition through PI3K/AKT pathway in gastric cancer. Int J Clin Exp Pathol 7: 7286-7296, 2014.

28. Zhang L, Zhao H, Zhang X, Chen L, Zhao X, Bai X and Zhang J: Nobiletin protects against cerebral ischemia via activating the p-Akt, p-CREB, BDNF and Bcl-2 pathway and ameliorating BBB permeability in rat. Brain Res Bull 96: 45-53, 2013.

29. Malik S, Bhatia J, Suchal K, Gamad N, Dinda AK, Gupta YK and Arya DS: Nobiletin ameliorates cisplatin-induced acute kidney injury due to its anti-oxidant, anti-inflammatory and anti-apoptotic effects. Exp Toxicol Pathol 67: 427-433, 2015.

30. Alijanpour S, Rezayof A, Sepehri H and Delphi L: Alterations in the hippocampal phosphorylated CREB expression in drug state-dependent learning. Behav Brain Res 292: 109-115, 2015.

31. Ortega-Martinez S: A new perspective on the role of the CREB family of transcription factors in memory consolidation via adult hippocampal neurogenesis. Front Mol Neurosci 8: 46, 2015

32. Wu L, Zhao QS, Li TW, et al: Yifei Xuanfei Jiangzhuo formula, a Chinese herbal decoction, improves memory impairment through inhibiting apoptosis and enhancing PKA/CREB signal transduction in rats with cerebral ischemia/reperfusion. Mol Med Rep 12: 4273-4283, 2015.

33. Aguiar AS Jr, Castro AA, Moreira EL, Glaser V, Santos AR, Tasca CI, Latini A and Prediger RD: Short bouts of mild-intensity physical exercise improve spatial learning and memory in aging rats: Involvement of hippocampal plasticity via AKT, CREB and BDNF signaling. Mech Ageing Dev 132: 560-567, 2011.

34. Zhang Y, Lan R, Wang J, Li XY, Zhu DN, Ma YZ, Wu JT and Liu ZH: Acupuncture reduced apoptosis and up-regulated BDNF and GDNF expression in hippocampus following hypoxia-ischemia in neonatal rats. J Ethnopharmacol 172: 124-132, 2015

35. Zhang F, Zhu ZQ, Liu DX, Zhang C, Gong QH and Zhu YH: Emulsified isoflurane anesthesia decreases brain-derived neurotrophic factor expression and induces cognitive dysfunction in adult rats. Exp Ther Med 8: 471-477, 2014.

36. Rak K, Völker J, Frenz S, Scherzad A, Schendzielorz P, Radeloff A, Jablonka S, Hagen R and Mlynski R: Effects of the neurotrophic factors BDNF, NT-3, and FGF2 on dissociated neurons of the cochlear nucleus. Neuroreport 25: 960-964, 2014.

37. Li J, Zhou Y, Liu BB, Liu Q, Geng D, Weng LJ and Yi LT: Nobiletin ameliorates the deficits in Hippocampal BDNF, TrkB and Synapsin I induced by chronic unpredictable mild stress. Evid Based Complement Alternat Med 2013: 359682, 2013. 